\title{
Fibrocyte-Like Cells from Intrauterine Growth Restriction Placentas Have a Reduced Ability to Stimulate Angiogenesis
}

\author{
Meghan R. Riddell, ${ }^{*}$ Bonnie Winkler-Lowen, ${ }^{\dagger}$ Yanyan Jiang, ${ }^{\ddagger}$ Larry J. Guilbert, ${ }^{\dagger}$ and Sandra T. Davidge ${ }^{\star \ddagger}$ \\ From the Departments of Physiology, ${ }^{*}$ Medical Microbiology and Immunology, ${ }^{\dagger}$ and Obstetrics and Gynecology, ${ }^{\ddagger}$ University of Alberta, Edmonton, Alberta, \\ Canada
}

\author{
Accepted for publication \\ June 4, 2013 \\ Address correspondence to \\ Sandra T. Davidge, Ph.D., 232 \\ HMRC, University of Alberta, \\ Edmonton, AB, Canada T6G \\ 2S2. E-mail: sandra.davidge@ \\ ualberta.ca.
}

\begin{abstract}
Intrauterine growth restriction (IUGR) is a common complication of pregnancy whereby the fetus fails to achieve its genetic growth potential. Malformation of the placental vasculature is observed in IUGR and may be due to the development of the placenta in a chronically hypoxic environment. Recently, we identified that the predominant stromal cells in the angiogenic zones of the placenta are fibrocyte-like cells. The conditioned medium from fibrocyte-like cells (FcCM) has been shown to stimulate angiogenesis in vitro. Thus, we hypothesized that FCCM from IUGR cells would have a reduced ability to stimulate angiogenesis and that chronic hypoxia would decrease the ability of both normal and IUGR fibrocyte-like cells to stimulate angiogenesis. IUGR FCCM had a reduced ability to stimulate endothelial tubule-like structure formation and an increased ability to stimulate endothelial migration compared with normal FCCM. However, normal and IUGR FCCM produced in chronic hypoxia did not alter endothelial proliferation, migration, or tubule-like structure formation. IUGR FCCM was found to have reduced levels of the pro-angiogenic cytokine IL- 8 and increased levels of the anti-angiogenic factors activin-A and pigment epithelium-derived growth factor. Thus, alterations in the ability of IUGR fibrocyte-like cells to stimulate angiogenesis may contribute to the development of vascular malformation in IUGR, but in vitro these changes cannot be attributed to a chronically hypoxic environment. (Am J Pathol 2013, 183: 1025-1033; http://dx.doi.org/10.1016/j.ajpath.2013.06.007)
\end{abstract}

It is generally accepted that fetal and placental weights are directly related. This relation has primarily been established because of experimental reduction of placental size in sheep, but it can be clearly evidenced in the common human pregnancy complication of intrauterine growth restriction (IUGR). ${ }^{1,2}$ In IUGR, a fetus fails to achieve its genetic growth potential for a given gestational age and is therefore pathologically small. ${ }^{3}$ The placental weight of infants born IUGR has been shown to be reduced by $24 \%$ compared with infants with growth appropriate for gestational age, and the actual placental size of infants with IUGR has been shown to be reduced compared with infants appropriate for gestational age of the same birth weight. ${ }^{1}$

Two essential processes control the overall growth of the placenta and thus the growth of the fetus: the expansion of the trophoblastic epithelium via a process of differentiation and the extension of the placental vasculature by angiogenesis. IUGR is associated with alterations in both trophoblast differentiation and placental angiogenesis. ${ }^{4-8}$ Profound alterations in the structure of the terminal villous vasculature have been observed in IUGR placentas. A reduction in vascular branching and elongation of the terminal villous vasculature specifically in cases of IUGR with absent or reversed end-diastolic (ARED) umbilical artery waveforms has been documented in perfusion fixed tissue by electron microscopy. ${ }^{9}$ Peripheral villous (intermediate and terminal villi) capillary volumes, surface area,

\footnotetext{
Supported by a grant from the Canadian Institute for Health Research (L.J.G. and S.T.D.). S.T.D. is a Canada Research Chair (Tier 1) in Women's Cardiovascular Health and an Alberta Innovates - Health Solutions/Alberta Heritage Foundation for Medical Research Scientist. M.R.R. was supported by studentships from Albert Innovates-Health Solutions and the Women and Children's Health Research Institute/Mazankowski Heart Institute.
} 
and lengths have also been found to be decreased in multiple sterological studies. ${ }^{10-13}$ Both an increase (in cases of IUGR with persistent end-diastolic umbilical artery flow) and decrease (in cases of IUGR with ARED) in peripheral villous capillary branching have been observed with stereology. ${ }^{14}$ In addition, reduced endothelial and stromal cell proliferation has been documented in IUGR tissue. ${ }^{15}$ Thus, IUGR is associated with significant alterations in angiogenesis that manifest as structural alterations in the vasculature, but the mechanisms through which these alterations occur remain to be elucidated.

One of the predominant hypotheses on the etiology of IUGR is that it is caused by insufficient trophoblastic remodeling of the maternal spiral arteries during the first and early-second trimester that in turn reduces maternal blood supply to the developing fetoplacental unit. This reduction in oxygen delivery to the placenta is hypothesized to result in the morphological alterations observed in the established disease. Paradoxically, hypoxia is a well-established inducer of angiogenesis. Hypoxia stimulates the induction of important pro-angiogenic factors, such as vascular endothelial growth factor (VEGF)-A, through the hypoxiainducible factor (HIF) pathway. ${ }^{16}$ This ultimately stimulates angiogenesis to occur to increase the oxygen supply to an area. Once sufficient angiogenesis has occurred and tissue hypoxia has abated, the vasculature returns to its normally quiescent state. It has been hypothesized, based on the differences in terminal villous morphology observed in cases of IUGR with ARED versus IUGR with persistent end-diastolic umbilical artery flow, that instead of being hypoxic the intervillous space in IUGR with ARED is actually hyperoxic. ${ }^{17,18}$ An alternative hypothesis that we are addressing is that long-term chronic hypoxia could result in the reduced angiogenesis observed in IUGR and that it is the chronic nature of the insult that leads to reduced angiogenesis. Angiogenesis is clearly deficient in IUGR placentas, but, because placental tissue cannot be collected until IUGR is already established, the effects of hypoxia on placental angiogenesis over time are unknown. Thus, experiments that examine whether a hypoxic environment can result in the altered morphology ultimately observed are required. Whether hypoxia may initially stimulate placental angiogenesis early on in the progression of IUGR, but has the opposite effect after chronic long-term exposure resulting in the reduced angiogenesis observed by the time of delivery, or whether the placentas of fetuses that develop IUGR do not respond in similar ways as those from a fetus that achieves normal growth are important questions to address. We hypothesize that chronic long-term unresolved hypoxia would ultimately result in cessation of angiogenesis due to a focus on cell survival in the suboptimal environment instead of sustaining the classical pro-angiogenic response for prolonged periods. This is most likely to manifest as a change in the balance of pro- and antiangiogenic factors after chronic hypoxic exposure (days to weeks) and could manifest differently in the different cellular constituents of the placenta and result in the overall decreased angiogenesis that has been repeatedly observed in IUGR placentas.

The cell types that are involved in stimulating placental angiogenesis are also largely unknown. The endothelium itself has a key role in integrating and responding to microenvironmental signals but other cellular components within the placenta likely play important roles through physical interaction with the endothelium and through the production of secreted pro- and anti-angiogenic factors. The predominant site of angiogenesis within the placenta is at the level of the terminal villi. ${ }^{19}$ This villous type is dominated by the vasculature, with $>50 \%$ of the villous volume taken up by blood vessels. ${ }^{20,21}$ The endothelium is therefore in close proximity to the epithelial trophoblast layer, which undoubtedly has a role in controlling angiogenesis, although the mechanisms remain to be defined. We have recently shown that fibrocyte-like cells are the predominant type of fibroblastic stromal constituent within the terminal villi. ${ }^{22}$ Moreover, isolated fibrocyte-like cell conditioned medium $(\mathrm{FcCM})$ is capable of stimulating placental endothelial cell (PEC) angiogenesis in vitro. Thus, this cell type is also likely to play an important role in placental angiogenesis.

We were therefore interested to understand whether fibrocyte-like cells from IUGR placentas have the same capacity to stimulate angiogenesis as those from a normal placenta and whether maintenance in chronic hypoxia would affect the ability of both normal and IUGR fibrocytelike cells to stimulate angiogenesis. We hypothesized that FcCM from IUGR placentas would have decreased ability to stimulate angiogenesis in vitro and that maintenance of both normal and IUGR fibrocyte-like cells in chronic hypoxia would result in a decrease in their ability to stimulate angiogenesis.

\section{Materials and Methods}

\section{Tissue Collection}

All placentas were collected from the Royal Alexandra Hospital in Edmonton, AB, Canada after proper ethical approval from the University of Alberta Ethics Committee, and informed consent was obtained. Normal control samples were collected from singleton uncomplicated term pregnancies. IUGR samples were collected from pregnancies in which the fetus had a decrease in growth trajectory documented by serial ultrasound assessment either in the presence or absence of an asymmetric growth pattern. IUGR samples were also confirmed to be negative for toxoplasmosis, other (agents), rubella, cytomegalovirus, and herpes (simplex) and to have a predicted fetal weight by ultrasound scanning that fell below the 10th percentile. All placentas were transported to the laboratory, and isolation of cells was started within 1 hour of birth. Normal term placental samples were used as a control population to eliminate the potentially confounding effects of other causes of 
prematurity. Table 1 presents the patient characteristics of all normal and IUGR placentas used in the study.

\section{Fibrocyte-Like Cell Isolation}

Fibrocyte-like cells, which are fibroblastic peripheral villi stromal constituents that express a combination of hematopoietic and fibroblastic markers, were isolated from both normal and IUGR placentas as previously described. ${ }^{22}$ Briefly, placental tissue was rinsed extensively to remove visible blood, and minced tissue was subjected to serial trypsin-DNase digestion, followed by adherence selection and $\mathrm{CD} 45^{+}$cell sorting. This method results in a population of morphologically fibroblastic cells that express the monocyte/macrophage markers CD14 and CD115 as well as the fibroblastic marker TE-7 at purities exceeding 95\%. Although the cells are initially selected on the basis of CD45 positivity, CD45 expression is lost over time in culture.

\section{PEC Isolation}

PECs were isolated from normal term placentas as previously described. ${ }^{22}$ Briefly, isolated placental vasculature was serially digested with a trypsin-pronase solution, followed by CD31 selection with magnetic beads (CD31 Dynal beads; Invitrogen, Carlsbad, CA). Cells were then plated in an atmosphere of $6 \% \mathrm{O}_{2} 5 \% \mathrm{CO}_{2}$, atmospheric conditions considered physiological for placental cells, ${ }^{23,24}$ in EGM-2MV (containing complete supplements; Lonza, Walkersville, MD). After a single passage cells were reselected with CD31 magnetic beads. PEC cultures were then maintained at $6 \% \mathrm{O}_{2} 5 \% \mathrm{CO}_{2}$, and this oxygen content was found to be essential for PEC growth, because isolation was not successful when the cells were maintained at higher oxygen concentrations ( $8 \%$ to $21 \% \mathrm{O}_{2}$ ).

\section{Production of FcCM}

Fibrocyte-like cells were maintained at either a fully humidified atmosphere of $6 \% \mathrm{O}_{2} 5 \% \mathrm{CO}_{2}$ (normoxia) or $1 \%$ $\mathrm{O}_{2} 5 \% \mathrm{CO}_{2}$ (hypoxia) in a Forma model 3130 incubator (Marietta, $\mathrm{OH}$ ) at $37^{\circ} \mathrm{C}$ and were maintained for a complete passage under these atmospheric conditions before conditioned medium was collected (approximately 1 week). Conditioned medium was collected as detailed in Riddell et al. $^{22}$ Fibrocyte-like cells were used to make FcCM between passages 2 to 8 .

\section{Endothelial Cell Migration Assay}

PECs were trypsinized, and 80,000 cells were seeded in endothelial basal medium (EBM)- $2+0.2 \%$ bovine serum albumin (supplement-free basal endothelial cell medium) in the top of a human fibronectin-coated transwell insert (12well plate; $3-\mu \mathrm{m}$ pore size; Becton Dickinson, Bedford, MA). FcCM or EBM-2 was made $2 \%$ with fetal calf serum (FCS), antibiotics were added, and then the medium was placed in the bottom of each well alongside an EGM$2 \mathrm{MV}-$ positive control. The plate was incubated at $6 \% \mathrm{O}_{2}$ $5 \% \mathrm{CO}_{2}$ for 24 hours. Inserts were then fixed in methanol, cells remaining on the top of the insert were removed with cotton swabs, and inserts were then stained with hematoxylin. The total number of cells that had migrated to the bottom side of the insert was manually counted for the entire

Table 1 Patient Characteristics

\begin{tabular}{|c|c|c|c|c|c|c|c|c|c|c|c|}
\hline Patient & $\begin{array}{l}\text { Fetal } \\
\text { diagnosis }\end{array}$ & $\begin{array}{l}\text { Birth } \\
\text { weight }(\mathrm{g})\end{array}$ & $\begin{array}{l}\text { Birth weight } \\
\text { percentile }\end{array}$ & $\begin{array}{l}\text { Gestational } \\
\text { Age (weeks) }\end{array}$ & $\begin{array}{l}\text { Umbilical } \\
\text { artery } \\
\text { wave form }\end{array}$ & $\begin{array}{l}\text { Maternal } \\
\text { complications }\end{array}$ & Sex & $\begin{array}{l}\text { Delivery } \\
\text { method }\end{array}$ & $\begin{array}{l}\text { Maternal } \\
\text { age (years) }\end{array}$ & Gravidity & $\begin{array}{l}\text { Placental } \\
\text { weight }(\mathrm{g})\end{array}$ \\
\hline 2 & IUGR & 700 & $5-10$ & 27.14 & ARED & & $M$ & Cs & 28 & 2 & 102 \\
\hline 3 & IUGR & 1590 & $<3$ & 35.14 & PED & & $M$ & Cs & 33 & 2 & 269 \\
\hline 4 & IUGR & 430 & $<3$ & 24.57 & ARED & PE, HELLP & $M$ & Cs & 22 & 1 & 156 \\
\hline 7 & IUGR & 1070 & $<3$ & 32.14 & NR & & $M$ & Cs & 24 & 1 & 187 \\
\hline 8 & Norm & 3280 & $10-50$ & 39.14 & NR & & $M$ & Cs & 36 & 3 & NR \\
\hline 9 & Norm & 3670 & $50-90$ & 39.00 & NR & & $M$ & Cs & 28 & 2 & NR \\
\hline 10 & Norm & 3290 & $50-90$ & 38.43 & NR & & $\mathrm{F}$ & Cs & 42 & 3 & NR \\
\hline 11 & Norm & 3430 & $50-90$ & 39.57 & NR & & $M$ & Cs & 32 & 2 & NR \\
\hline 16 & Norm & 3170 & $50-90$ & 38.57 & NR & & $M$ & Cs & 34 & 5 & NR \\
\hline 17 & Norm & 3090 & $50-90$ & 38.14 & NR & & $M$ & Cs & 30 & 4 & NR \\
\hline
\end{tabular}

AED, absent end-diastolic flow; ARED, absent or reversed end-diastolic flow; $\mathrm{Cs}$, caesarean section; HELLP, hemolysis, elevated liver enzymes, low platelets syndrome; IUGR, intrauterine growth restriction; Norm, normal; NR, not reported; PE, preeclampsia; PED, persistent end-diastolic flow; Vd, vaginal delivery. 
insert. Each treatment group was normalized to the EBM$2-$ negative control, and the experiment was repeated three separate times with the same conditioned medium ( $n=5$ to 6) on PECs isolated from three patients. The relative value for each conditioned medium sample obtained in the separate assay runs was then averaged and are displayed in the figures.

\section{Endothelial Cell Proliferation Assay}

PECs were trypsinized, and 10,000 cells per well were seeded in 96-well plates in quadruplicate for each treatment in EBM-2 $+0.5 \%$ FCS (basal endothelial medium) and incubated for 4 hours at $6 \% \mathrm{O}_{2} 5 \% \mathrm{CO}_{2}$. After 4 hours a single well from each treatment group was fixed with methanol for 10 minutes to serve as a time 0 baseline cell number. Meanwhile medium was changed to $\mathrm{FcCM}$ supplemented with $0.5 \%$ FCS and antibiotics, EBM- $2+0.5 \%$ FCS (negative control), or EGM-2MV (positive control) in all remaining wells (in triplicate). Cells were then incubated for 48 hours. All remaining wells were then fixed with methanol, and all wells including the time 0 control were stained with DRAQ5 (Biostatus, Leicestershire, UK) for 1 hour. The entire plate was then scanned on the Licor Odyssey (Guelph, ON, Canada). Integrated intensity values obtained for the time 0 baseline cell number wells were averaged, and all other wells were normalized to this baseline cell number to represent the relative number of cells compared with time 0 . Values were normalized to the EBM-2-negative control, and proliferation assays were repeated with conditioned medium collected from the same placentas ( $n=4$ to 5 for each group) three separate times on PECs isolated from three patients. The relative value for each conditioned medium sample obtained in the separate assay runs was then averaged and is displayed in the figures.

\section{Endothelial Cell Tubule-Like Structure Formation Assay}

Growth factor-reduced Matrigel (GFR-Matrigel; Becton Dickinson) was thawed overnight on ice and plated at $45 \mu \mathrm{L}$ per well in a 96-well plate. The plate was then incubated for 30 minutes at $6 \% \mathrm{O}_{2} 5 \% \mathrm{CO}_{2}$. Meanwhile, PECs were trypsinized and resuspended in EBM-2 $+1 \%$ FCS with antibiotics, and 10,000 cells per well were then seeded on the solidified GFR-Matrigel in triplicate for each treatment. The plate was then placed back into the incubator for 1 hour. Conditioned medium or EBM-2 was made to $1 \%$ FCS plus antibiotics, and after 1 hour experimental medium, including an EGM-2MV-positive control, was added. The plate was incubated for 6 hours at $6 \% \mathrm{O}_{2} 5 \% \mathrm{CO}_{2}$, and all wells were then fixed with $4 \%$ paraformaldehyde. Duplicate images were captured for each well at $5 \times$ magnification on a Leica DMIRB microscope (Wetzlar, Germany) equipped with a QImaging Retiga EX camera (Surrey, BD, Canada) with the use of Openlab software version 4.0.2 (PerkinElmer, Waltham, MA). Images were analyzed with ImageJ software version $1.43 \mathrm{u}(\mathrm{NIH}$, Bethesda, MD), and total tubule-like structure number was counted and length of each tubule was measured. These values were then normalized to the EBM-2 + 1\% FCS-negative control for each assay run. The assay was repeated three separate times with the same conditioned medium ( $n=3$ to 4$)$ on PECs isolated from three patients. The relative value for each conditioned medium sample obtained in the separate assay runs was then averaged and is displayed in the figures.

\section{Angiogenic Factor Proteome Array}

As a way to generate hypotheses about which pro- and antiangiogenic factors were differentially regulated in fibrocytelike cells from normal and IUGR placentas and under different oxygen conditions, $\mathrm{FcCM}$ was produced, using the same methods presented earlier, from a single healthy patient (patient 10) and a single patient with IUGR (patient 2). FcCM from these patients produced under normoxia and chronic hypoxia were assessed in a proteome profiler human angiogenesis array (R\&D Systems, Minneapolis, MN) according to the manufacturer's instructions. Data were obtained on the presence of 43 different pro- and anti-angiogenic factors, and the relative expression in each group was compared. See Supplemental Table S1 for the relative protein expression of observed angiogenic factors.

\section{Measurement of Pro- and Anti-Angiogenic Growth Factors}

Specific growth factors were selected for further investigation on the basis of an observed differential expression pattern in the proteome array or a known importance in placental angiogenesis from the literature. Growth factor concentrations were measured by ELISA for IL-8 (RayBiotech, Norcross, GA), endocrine gland (EG)-VEGF, activin-A, and VEGF-A (R\&D Systems) in six normal $\mathrm{FcCM}$ and five IUGR FcCM serum free. Results were normalized to the total protein content of the wells from which the medium was collected. Protein concentrations were measured by the bicinchoninic acid protein assay. IL-8 levels of four normal $\mathrm{FcCM}$ samples were found to be above detection limits for the assay despite 1:4 dilution; thus, values for these samples were represented as the highest detectable level for the assay and normalized as for the other samples.

Pigment epithelial-derived factor (PEDF) and soluble Flt-1 (sFlt-1) levels were determined by Western blot analysis of concentrated conditioned medium. Centrifuged and filtered conditioned medium was immediately concentrated by using Amicon Ultra centrifugal units $(30-\mathrm{kDa}$ cutoff; Millipore, Billerica, MA) and frozen at $-80^{\circ} \mathrm{C}$ until needed. Twenty-four micrograms of protein was loaded onto a $9 \%$ SDS-PAGE gel (PEDF) or a 6\% SDS-PAGE gel (sFlt-1) and then transferred onto a nitrocellulose membrane. The blots were then probed with anti-PEDF (Santa Cruz 
Biotechnology, Santa Cruz, CA) or anti-Flt-1 (R\&D Systems) overnight at $4^{\circ} \mathrm{C}$. After repeated washes the blot was probed with anti-goat far fluorescent-tagged secondary antibody for 1 hour at room temperature. After repeated washes the blots were visualized by using the Licor Odyssey. PEDF and sFlt-1 were normalized to a running standard control that was run on each blot and corrected by the concentration factor and to the total amount of protein in the wells in which the conditioned medium was produced.

\section{IL-8 and Activin-A Tubule-Like Structure Dose Response}

To assess whether direct effects of the different concentrations of IL-8 and activin-A found in IUGR versus normal FcCM influence PEC tubule-like structure formation, endothelial cell tubule-like structure formation assays were performed. Tubule-like structure formation assays were set up and analyzed as presented above. For the IL- 8 assays the experimental groups comprise an EBM-2-negative control and IL-8 doses that represented the average concentration found in IUGR FcCM $(100 \mathrm{pg} / \mathrm{mL})$, the average concentration found in normal FcCM $(300 \mathrm{pg} / \mathrm{mL})$, and the two highest concentrations found in normal FcCM (900 and $1200 \mathrm{pg} / \mathrm{mL}$ ).

For the activin-A assays, the experimental groups comprise an EBM-2-negative control, a VEGF-positive control $(10 \mathrm{ng} / \mathrm{mL})$, and VEGF (10 ng/mL) plus activin-A treatments that represent the average concentration of activin-A found in normal FcCM $(300 \mathrm{pg} / \mathrm{mL})$, a lower dose representative of lower-end concentrations in normal FcCM $(100 \mathrm{pg} / \mathrm{mL})$, the average concentration found in IUGR FcCM $(1000 \mathrm{pg} / \mathrm{mL})$, and the highest concentrations found in IUGR FcCM $(1300 \mathrm{pg} / \mathrm{mL})$.

\section{Statistics}

Statistical tests used for individual experiments are detailed in the figure legends. A two-way analysis of variance with a Bonferroni post hoc test was used to analyze the angiogenesis assays and to identify differences in growth factor expression between the groups. All statistics were performed with Prism software version 5.0 (GraphPad Software, San Diego, CA), and $P<0.05$ was considered significant.

\section{Results}

Fibrocyte-like cells were isolated from placentas collected from seven pregnancies complicated with IUGR and 10 normal pregnancies. Patient characteristics for all collected samples are presented in Table 1.

FcCM produced under all conditions stimulated PEC migration (Figure 1A). Chronic hypoxia did not have a significant effect on PEC migration in normal or IUGR FcCM, but IUGR FcCM stimulated significantly more migration than control. In contrast, FcCM collected from both normal and IUGR fibrocyte-like cells under normoxia
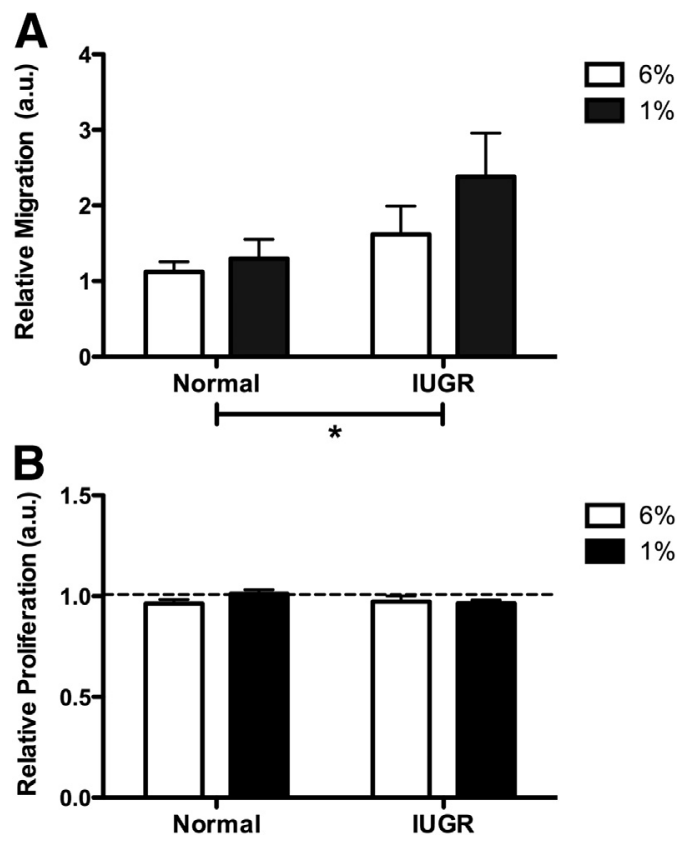

Figure 1 Fibrocyte-like cell conditioned medium (FCCM) collected from intrauterine growth restriction (IUGR) fibrocyte-like cells stimulates increased placental endothelial cell (PEC) migration compared with normal FCCM but fails to stimulate PEC proliferation. A: PEC migration; means \pm SEM; two-way analysis of variance with Bonferroni post hoc analysis; $n=5$ to 6. a.u., arbitrary units. ${ }^{*} P<0.05$. B: PEC proliferation; means $\pm \mathrm{SEM}$; $n=4$ to 5 .

and chronic hypoxia failed to stimulate PEC proliferation (Figure 1B).

FcCM has previously been shown to stimulated PEC and human umbilical vein endothelial cell tubule-like structure formation on GFR-Matrigel. ${ }^{22}$ FcCM produced by IUGR fibrocyte-like cells stimulated formation of approximately $22 \%$ fewer tubule-like structures than FcCM from normal fibrocyte-like cells (Figure 2, A, C, and D). IUGR-FcCM also resulted in an increased tubule-like structure length by approximately $7 \%$ compared with normal FcCM (Figure 2B). Thus, IUGR-FcCM stimulated the formation of significantly fewer but longer tubules overall. No difference was observed in the number of tubule-like structures or the tubule-like structure length when PECs were treated with FcCM produced under chronic hypoxia.

The amount of the pro-angiogenic factors IL-8, VEGF-A, and EG-VEGF in FcCM were examined by ELISA. IL-8 was found to be significantly lower in the FcCM from IUGR samples (threefold to sevenfold lower), but no effect of chronic hypoxia was observed (Figure 3A). In addition, IL-8 doses in the concentration range found in both normal and IUGR FcCM were unable to stimulate significant tubule-like structure formation (Supplemental Figure S1). VEGF-A levels were not significantly different between normal and IUGR FcCM, but chronic hypoxia was found to result in a threefold increase in VEGF-A compared with FcCM produced under normoxia (Figure 3B). No differences were found between any of the groups for EG-VEGF (Figure 3C). 
A

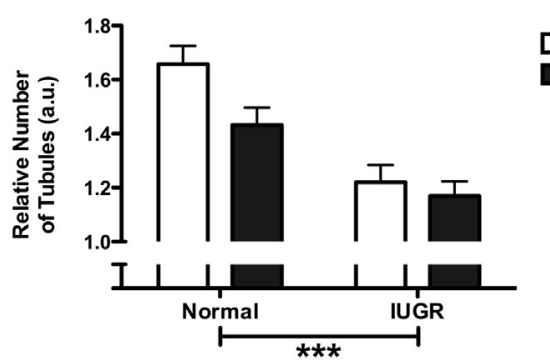

B

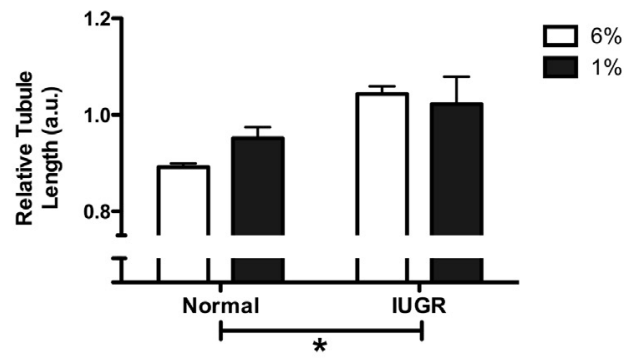

C

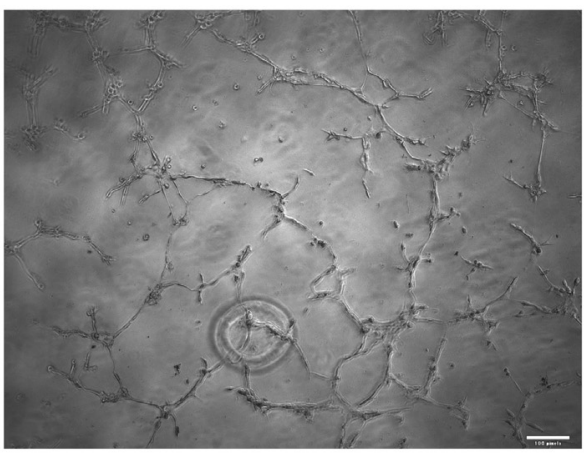

D

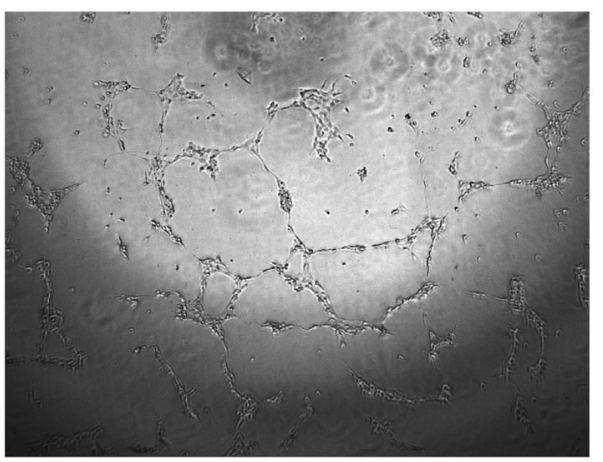

Figure 2 Intrauterine growth restriction (IUGR) fibrocyte-like cell conditioned medium ( $\mathrm{FCCM}$ ) has a reduced ability to stimulate placental endothelial cell (PEC) tubule-like structure formation. A: Summary graph of relative number of tubule-like structures. B: Summary graph of relative tubule-like structure length; means $\pm S E M$; two-way analysis of variance with Bonferroni post hoc analysis; $n=3$ to 4 . a.u., arbitrary units. ${ }^{*} P<$ $0.05, * * * P<0.001$. Representative images of tubule-like structures with normal FcCM $6 \% \mathrm{O}_{2}$ (C) or IUGR FCCM $6 \% 0_{2}$ (D). Scale bar $=50 \mu \mathrm{m}$.

In addition, no correlation was found between gestational age and the production of IL-8, VEGF-A, or EG-VEGF in FcCM.

The anti-angiogenic factors activin-A, PEDF, and sFlt-1 were also examined. Activin-A was found to be approximately threefold higher by ELISA in IUGR FcCM, but no effect of chronic hypoxia was observed (Figure 4A). In addition, no correlation was found between gestational age and the production of activin- $\mathrm{A}$, and activin-A treatment in the concentration range observed in normal and IUGR FcCM was not able to significantly inhibit VEGF-stimulated tubulelike structure formation (Supplemental Figure S2). PEDF was also found to be in approximately fivefold higher abundance in IUGR FcCM at $6 \% \mathrm{O}_{2}$ and was found in significantly decreased levels (approximately fivefold to eightfold) in $\mathrm{FcCM}$ produced under chronic hypoxia by Western blot analysis (Figure 4B). Chronic hypoxia increased the levels of sFlt- 1 found in FcCM by Western blot analysis by approximately threefold, but no difference was found in sFlt-1 levels between normal and IUGR FcCM (Figure 4C).

\section{Discussion}

In this study we have found that $\mathrm{FcCM}$ from IUGR placentas have a reduced ability to stimulate endothelial cell
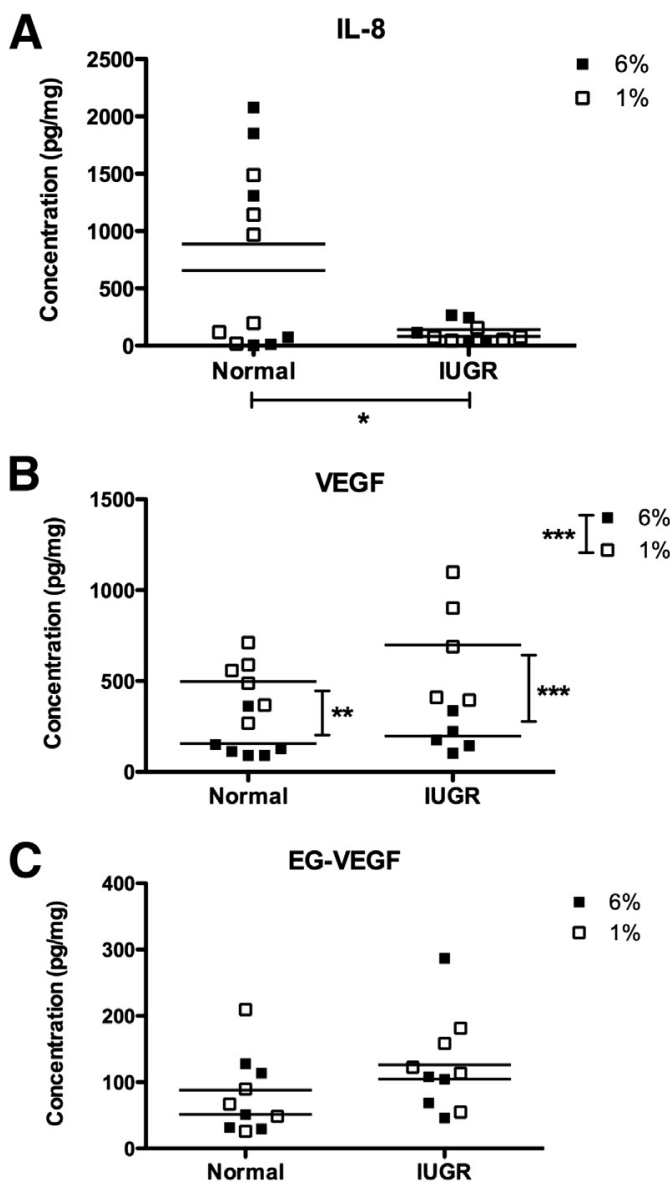

Figure 3 Fibrocyte-like cell conditioned medium (FCCM) from intrauterine growth restriction (IUGR) fibrocyte-like cells has decreased levels of pro-angiogenic factors compared with normal FcCM. A: Summary graph of IL-8 measured by ELISA; two-way analysis of variance with Bonferroni post hoc analysis; line represents mean; $n=5$ to 6 . ${ }^{*} P<0.05$. B: Summary graph of vascular endothelial growth factor-A (VEGF-A) measured by ELISA; $n=5$ to $6 .{ }^{*} P<0.01,{ }^{* *} P<0.001$. C: Summary graph of endocrine gland-VEGF (EG-VEGF) measured by ELISA; $n=5$. 

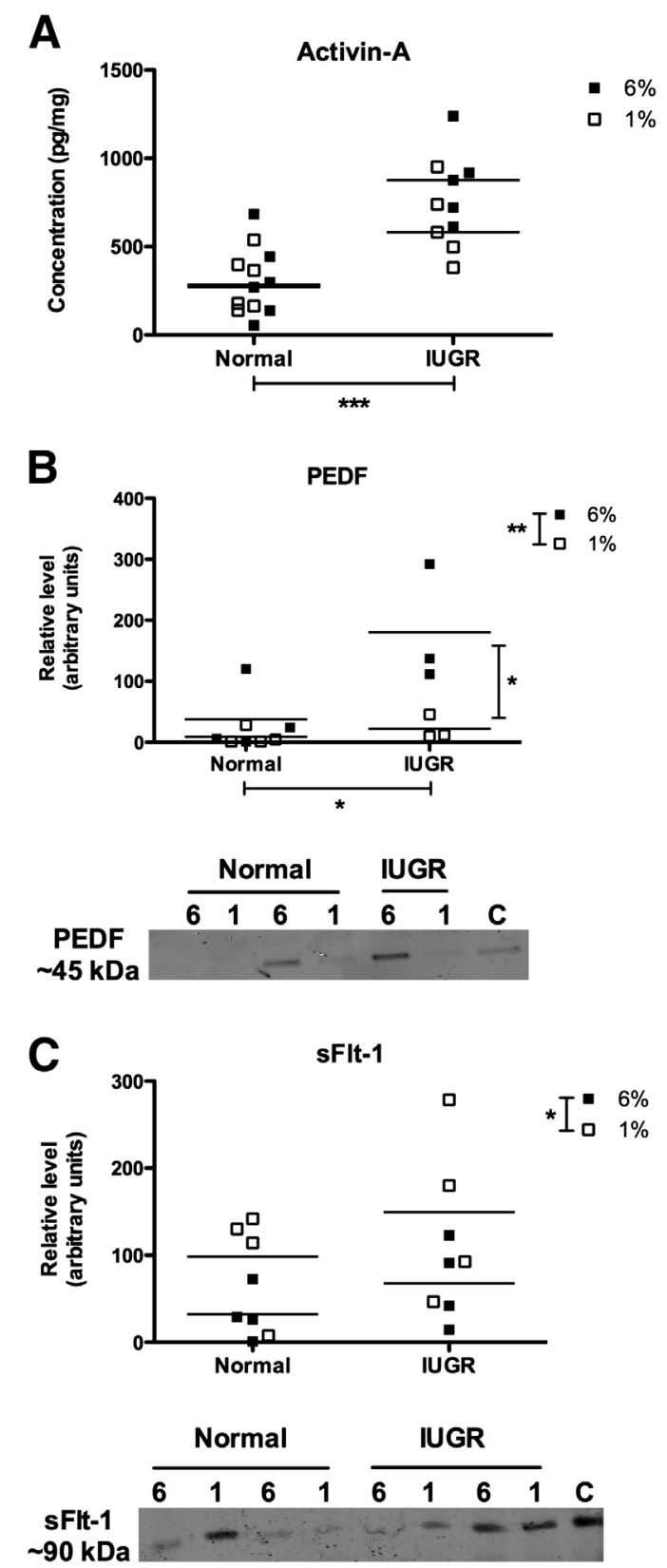

Figure 4 Fibrocyte-like cell conditioned medium ( $\mathrm{FcCM}$ ) from intrauterine growth restriction (IUGR) fibrocyte-like cells has increased levels of anti-angiogenic factors compared with normal FCCM. A: Summary graph of activin-A measured by ELISA; two-way analysis of variance with Bonferroni post hoc analysis; line represents mean; $n=5$ to $6 .{ }^{* *} P<0.001$. B: Summary graph of pigment epithelial-derived factor (PEDF) Western blot analysis and a representative Western blot; $n=3$ to 4 . ${ }^{*} P<0.05,{ }^{* *} P<$ 0.01 . C: Summary graph of sFlt- 1 Western blot analysis and a representative Western blot; $n=4$. ${ }^{*} P<0.05$. 6 indicates $6 \% 0_{2} ; 1,1 \% 0_{2} ; C$, loading control.

tubule-like structure formation and an increased ability to stimulate endothelial cell migration than fibrocyte-like cells from normal placentas. It was found that IUGR FcCM contained reduced levels of the pro-angiogenic factor IL-8 and increased levels of the anti-angiogenic factors activin-A and PEDF. Thus, fibrocyte-like cells isolated from IUGR placentas have an altered ability to stimulate angiogenesis in vitro. This implies that there are inherent differences in the behavior of the same cell type in placentas that result in reduced fetal growth and those that allow for adequate fetal growth. A difference in the ability of conditioned medium collected from placental explants from normal and IUGR placentas to stimulate angiogenesis in vitro has been previously shown by in placental explants. ${ }^{25}$ In contrast to our study Padavala et $\mathrm{al}^{25}$ did not see a difference in the ability of conditioned medium from IUGR explants to stimulate human umbilical vein endothelial cell tubule-like structure formation at high oxygen tension $\left(20 \% \mathrm{O}_{2}\right)$ but saw a reduction in the tubule-like structure length alone when conditioned medium was collected from explants cultured at $3 \% \mathrm{O}_{2}$. Thus, the differential ability of normal versus IUGR placental cells to stimulate angiogenesis required a low oxygen environment to be observed. In our study, specifically examining fibrocyte-like cells, IUGR FcCM stimulated formation of fewer tubule-like structures under both normoxia $\left(6 \% \mathrm{O}_{2}\right)$ and chronic hypoxia $\left(1 \% \mathrm{O}_{2}\right)$, and IUGR FcCM simulated an increased tubule-like structure length which was only significant at $6 \% \mathrm{O}_{2}$. Thus, both our study and others ${ }^{25}$ show that normal and IUGR placental cells have differential abilities to stimulate angiogenesis in vitro. Conditioned medium collected from placental explants is more representative of the soluble factors produced by the syncytiotrophoblast; therefore, it appears that a dysfunctional ability to stimulate angiogenesis is present in two different populations of cells that are involved in controlling placental angiogenesis.

A secondary goal of this study was to examine whether exposure to chronic hypoxia would result in a decreased ability of fibrocyte-like cells to stimulate angiogenesis in vitro irrespective of their isolation from normal or IUGR placentas. It was more specifically hypothesized that this would be due to an inability of the fibrocyte-like cells to produce a pro-angiogenic response to hypoxia when hypoxia was administered chronically. It was found that maintenance in chronic hypoxia did not result in any differential ability of FcCM to stimulate endothelial cell proliferation, migration, or differentiation into tubule-like structures; therefore, chronic hypoxia does not result in a decreased ability to stimulate any aspect of angiogenesis under these conditions. In addition, an increased amount of VEGF-A and sFlt-1 and a decreased amount of PEDF were found in conditioned medium produced under chronic hypoxia. Hypoxia is a known inducer of pro-angiogenic factors such as VEGF-A, stromal cell-derived factor 1 , endothelin-1, IL-8, and EG-VEGF and the anti-angiogenic factor sFlt-1. ${ }^{26,27}$ In this study chronic hypoxia led to the elevation of both VEGF-A and sFlt-1. This implies that the HIF pathway is activated despite the chronic nature of the insult, because both of these factors are known to be regulated by HIF. ${ }^{26}$ Up-regulation of IL-8 and EG-VEGF reported in the literature is independent of the HIF pathway, ${ }^{26}$ and no effect of chronic hypoxia was found on the levels of 
either factor in our study. In this study PEDF production was also found to be controlled by hypoxia, with a reduced amount of PEDF found in FcCM produced under chronic hypoxia. Hypoxia has previously been shown to both increase and decrease PEDF expression, depending on the cell type, and its regulation has been shown to be both HIF dependent and independent. ${ }^{28-30}$ Ultimately, in this study we found that, although there is a significant increase in VEGF-A after chronic hypoxic treatment, this increase is not able to influence PEC proliferation, migration, or tubulelike structure formation, likely because of the observed increase in sFlt-1 levels. Thus, the balance of factors found in the $\mathrm{FcCM}$ under chronic hypoxia is ultimately no more pro-angiogenic than FcCM produced under physiological normoxia.

To try to understand the mechanisms through which IUGR fibrocyte-like cells were stimulating the reduced formation of tubule-like structure and increased PEC migration we examined the abundance of several pro- and anti-angiogenic growth factors in the conditioned medium. A significant reduction in the pro-angiogenic factor IL-8 and an increase in the anti-angiogenic factors activin-A and PEDF were found. When the pro- and anti-angiogenic abilities of IL-8 and activin A were examined by using the tubule-like structure formation assay and the concentration ranges of the factors observed in both normal and IUGR $\mathrm{FcCM}$, neither factor was able to significantly influence PEC tubule-like structure formation on GFR-Matrigel. These results indicate that it is unlikely that IL- 8 and activin $\mathrm{A}$ have dominant roles in controlling the tubule-like structure formation induced by FcCM. These observations highlight that the differential abilities of IUGR versus normal fibrocyte-like cells to stimulate angiogenesis are unlikely to be attributed to a single angiogenic factor but are, instead, likely a result of changes in multiple pro- and anti-angiogenic signals.

A potential pathway, which remains to be validated, that was identified by the proteome array and may be contributing to the observed increased PEC migration to IUGR $\mathrm{FcCM}$ is an increased expression of MCP-1. MCP-1 has been shown to directly stimulate endothelial cell migration at lower doses than it stimulates endothelial cell sprouting in the rat aortic ring assay $(500 \mathrm{pg} / \mathrm{mL}$ versus $50 \mathrm{ng} / \mathrm{mL}){ }^{31}$ Thus, if low levels of MCP-1 are present in the conditioned medium, it may be able to stimulate PEC migration without significantly contributing to tubule-like structure formation. Another observation that warrants further investigation is the lack of PEC proliferation when cells were treated with FcCM. Concentrated FcCM was also tested, but proliferation was not significantly improved (data not shown), thus ruling out the possibility that the concentration of growth factors in the conditioned medium was too low to stimulate proliferation. Therefore, it is likely that some factor or group of factors blocks proliferation. EGVEGF selectively acts on the placental microvascular endothelium to stimulate proliferation, migration, and differentiation, whereas it only stimulates macrovascular umbilical endothelial cell survival. ${ }^{32}$ In this study human umbilical vein endothelial cells failed to migrate toward $\mathrm{FcCM}$ and also failed to proliferate in the presence of $\mathrm{FcCM}$ (data not shown). Thus, there may be additional factors, in addition to EG-VEGF, in FcCM that may have placentalspecific angiogenic effects. Clear characterization of the components of $\mathrm{FcCM}$ could therefore illuminate previously unidentified factors that are contributing to the decreased angiogenesis stimulated by IUGR fibrocyte-like cells.

Ultimately, this communication has shown that there are inherent differences in the ability of normal versus IUGR fibrocyte-like cells to stimulate angiogenesis and that a chronically hypoxic environment is not able to differentially affect the ability of fibrocyte-like cells to stimulate angiogenesis in vitro. Thus, the differential production of angiogenic growth factors by fibrocyte-like cells may contribute to reduced placental growth and ultimately to the development of IUGR.

\section{Acknowledgment}

We thank our research nurse, Donna Dawson, for the collection of samples.

\section{Supplemental Data}

Supplemental material for this article can be found at http://dx.doi.org/10.1016/j.ajpath.2013.06.007.

\section{References}

1. Heinonen S, Taipale P, Saarikoski S: Weights of placentae from smallfor-gestational age infants revisited. Placenta 2001, 22:399-404

2. Alexander G: Studies on the placenta of the sheep (Ovis aries L.). Effect of surgical reduction in the number of caruncles. J Reprod Fertil 1964, 7:307-322

3. Rizzo G, Arduini D: Intrauterine growth restriction: diagnosis and management. A review. Minerva Ginecol 2009, 61:411-420

4. Ruebner M, Strissel PL, Langbein M, Fahlbusch F, Wachter DL, Faschingbauer F, Beckmann MW, Strick R: Impaired cell fusion and differentiation in placentae from patients with intrauterine growth restriction correlate with reduced levels of HERV envelope genes. J Mol Med (Berl) 2010, 88:1143-1156

5. Newhouse SM, Davidge ST, Winkler-Lowen B, Demianczuk N, Guilbert LJ: In vitro differentiation of villous trophoblasts from pregnancies complicated by intrauterine growth restriction with and without pre-eclampsia. Placenta 2007, 28:999-1003

6. Macara L, Kingdom JC, Kaufmann P, Kohnen G, Hair J, More IA, Lyall F, Greer IA: Structural analysis of placental terminal villi from growth-restricted pregnancies with abnormal umbilical artery Doppler waveforms. Placenta 1996, 17:37-48

7. Heazell AE, Sharp AN, Baker PN, Crocker IP: Intra-uterine growth restriction is associated with increased apoptosis and altered expression of proteins in the p53 pathway in villous trophoblast. Apoptosis 2011, $16: 135-144$

8. Dunk CE, Roggensack AM, Cox B, Perkins JE, Asenius F, Keating S, Weksberg R, Kingdom JC, Adamson SL: A distinct microvascular 
endothelial gene expression profile in severe IUGR placentas. Placenta 2012, 33:285-293

9. Krebs C, Macara LM, Leiser R, Bowman AW, Greer IA, Kingdom JC: Intrauterine growth restriction with absent end-diastolic flow velocity in the umbilical artery is associated with maldevelopment of the placental terminal villous tree. Am J Obstet Gynecol 1996, 175:1534-1542

10. Mayhew TM, Wijesekara J, Baker PN, Ong SS: Morphometric evidence that villous development and fetoplacental angiogenesis are compromised by intrauterine growth restriction but not by preeclampsia. Placenta 2004, 25:829-833

11. Egbor M, Ansari T, Morris N, Green CJ, Sibbons PD: Pre-eclampsia and fetal growth restriction: how morphometrically different is the placenta? Placenta 2006, 27:727-734

12. Egbor M, Ansari T, Morris N, Green CJ, Sibbons PD: Morphometric placental villous and vascular abnormalities in early- and late-onset pre-eclampsia with and without fetal growth restriction. BJOG 2006, 113:580-589

13. Mayhew TM, Ohadike C, Baker PN, Crocker IP, Mitchell C, Ong SS: Stereological investigation of placental morphology in pregnancies complicated by pre-eclampsia with and without intrauterine growth restriction. Placenta 2003, 24:219-226

14. Todros T, Sciarrone A, Piccoli E, Guiot C, Kaufmann P, Kingdom J: Umbilical Doppler waveforms and placental villous angiogenesis in pregnancies complicated by fetal growth restriction. Obstet Gynecol 1999, 93:499-503

15. Crocker IP, Tansinda DM, Baker PN: Altered cell kinetics in cultured placental villous explants in pregnancies complicated by pre-eclampsia and intrauterine growth restriction. J Pathol 2004, 204:11-18

16. Adams RH, Alitalo $\mathrm{K}$ : Molecular regulation of angiogenesis and lymphangiogenesis. Nat Rev Mol Cell Biol 2007, 8:464-478

17. Kingdom JC, Kaufmann P: Oxygen and placental villous development: origins of fetal hypoxia. Placenta 1997, 18:613-621; discussion 623-626

18. Mayhew TM, Charnock-Jones DS, Kaufmann P: Aspects of human fetoplacental vasculogenesis and angiogenesis, III: changes in complicated pregnancies. Placenta 2004, 25:127-139

19. Burton GJ, Charnock-Jones DS, Jauniaux E: Regulation of vascular growth and function in the human placenta. Reproduction 2009, 138: 895-902

20. Huppertz B: The anatomy of the normal placenta. J Clin Pathol 2008, 61:1296-1302
21. Jones CJ, Fox H: Ultrastructure of the normal human placenta. Electron Microsc Rev 1991, 4:129-178

22. Riddell MR, Winkler-Lowen B, Chakrabarti S, Dunk C, Davidge ST, Guilbert LJ: The characterization of fibrocyte-like cells: a novel fibroblastic cell of the placenta. Placenta 2012, 33:143-150

23. Soothill PW, Nicolaides $\mathrm{KH}$, Rodeck $\mathrm{CH}$, Campbell S: Effect of gestational age on fetal and intervillous blood gas and acid-base values in human pregnancy. Fetal Ther 1986, 1:168-175

24. Hung TH, Skepper JN, Burton GJ: In vitro ischemia-reperfusion injury in term human placenta as a model for oxidative stress in pathological pregnancies. Am J Pathol 2001, 159:1031-1043

25. Padavala S, Pope N, Baker P, Crocker I: An imbalance between vascular endothelial growth factor and its soluble receptor in placental villous explants of intrauterine growth-restricted pregnancies. J Soc Gynecol Investig 2006, 13:40-47

26. Fong GH: Mechanisms of adaptive angiogenesis to tissue hypoxia. Angiogenesis 2008, 11:121-140

27. Ahmad S, Ahmed A: Elevated placental soluble vascular endothelial growth factor receptor-1 inhibits angiogenesis in preeclampsia. Circ Res 2004, 95:884-891

28. Fernandez-Barral A, Orgaz JL, Gomez V, del Peso L, Calzada MJ, Jimenez B: Hypoxia negatively regulates antimetastatic PEDF in melanoma cells by a hypoxia inducible factor-independent, autophagy dependent mechanism. PLoS One 2012, 7:e32989

29. Yang XM, Yafai $Y$, Wiedemann $P$, Kuhrt H, Wang YS, Reichenbach A, Eichler W: Hypoxia-induced upregulation of pigment epithelium-derived factor by retinal glial (Muller) cells. J Neurosci Res 2012, 90:257-266

30. Rychli K, Kaun C, Hohensinner PJ, Dorfner AJ, Pfaffenberger S, Niessner A, Bauer M, Dietl W, Podesser BK, Maurer G, Huber K, Wojta J: The anti-angiogenic factor PEDF is present in the human heart and is regulated by anoxia in cardiac myocytes and fibroblasts. J Cell Mol Med 2010, 14:198-205

31. Salcedo R, Ponce ML, Young HA, Wasserman K, Ward JM, Kleinman HK, Oppenheim JJ, Murphy WJ: Human endothelial cells express CCR2 and respond to MCP-1: direct role of MCP-1 in angiogenesis and tumor progression. Blood 2000, 96:34-40

32. Brouillet S, Hoffmann P, Benharouga M, Salomon A, Schaal JP, Feige JJ, Alfaidy N: Molecular characterization of EG-VEGF-mediated angiogenesis: differential effects on microvascular and macrovascular endothelial cells. Mol Biol Cell 2010, 21:2832-2843 